\title{
Immunohistochemical study of parathyroid hormone-related protein in vesical transitional epithelium of patients with spinal cord injury
}

\author{
S Vaidyanathan*,1, DT McCreavy ${ }^{2}$, IW McDicken ${ }^{3}$, BM Soni ${ }^{1}$, P Mansour $^{4}$, B Wlodarski ${ }^{5}$, JA Carron ${ }^{5}$, \\ WD Fraser ${ }^{2}$, G Singh $^{1}$, P Sett ${ }^{1}$ and JA Gallagher ${ }^{5}$ \\ ${ }^{1}$ Regional Spinal Injuries Centre, District General Hospital, Southport, Merseyside PR8 6PN, UK; ${ }^{2}$ The Department \\ of Clinical Chemistry, The University of Liverpool, Liverpool L69 3GA, UK, ${ }^{3}$ Department of Pathology, The \\ University of Liverpool, Duncan Building, Liverpool L69 3GA, UK; ${ }^{4}$ Department of Histopathology, District General \\ Hospital, Southport, Merseyside PR8 6PN, UK, ${ }^{5}$ Department of Human Anatomy and Cell Biology, The University \\ of Liverpool, Liverpool L69 3GE, UK
}

\begin{abstract}
Introduction: Parathyroid hormone-related protein (PTHrP), in addition to the wellestablished role in endochrondral bone development, is believed to be an important mediator of cellular growth and differentiation in a number of non-bony tissues.

Objectives: To compare the immunohistochemical staining of vesical transitional epithelium to antibodies raised to synthetic peptides of PTHrP composed of amino acid sequences 43-52 and 127-138 in patients with spinal cord injury (SCI) and neuropathic bladder $(n=14)$, and control patients with intact neuraxis and no history of bladder cancer $(n=10)$.

Setting: Male SCI patients registered with Regional Spinal Injuries Centre, Southport, England.

Intervention: Endoscopic cold cup biopsy from the trigone of the urinary bladder was taken from patients with SCI while they were undergoing a therapeutic procedure in the urinary bladder. The control samples of bladder biopsies were taken from the archives of the Department of Histopathology, District General Hospital, Southport. Immunohistochemistry was performed using rabbit antibodies raised against synthetic peptides of human PTHrP (43-52) and PTHrP (127-138). The biopsies were examined for immunostaining of transitional epithelium.

Results: Of the 14 biopsies of SCI patients, positive immunostaining using antibodies to both the PTHrP peptides was found in four cases; five biopsies showed positive immunostaining only to anti-PTHrP (43-52); and five biopsies showed no immunostaining with either of the PTHrP peptides. In contrast, transitional epithelium in the biopsy specimens of ten control subjects with no history of bladder cancer showed no immunostaining with either of the PTHrP peptides.

Conclusion: This study revealed that the transitional epithelium of neuropathic urinary bladder exhibits increased predilection for positive immunohistochemical staining for PTHrP (43-52), and to a lesser extent, to PTHrP (127-138), as compared to the vesical transitional epithelium of able bodied individuals with no history of vesical malignancy. The possible role of PTHrP in the cellular differentiation of urothelium of neuropathic bladder, and thereby, in the pathogenesis of cystitis in SCI patients, needs to be explored.
\end{abstract}

Keywords: spinal cord injury; neuropathic bladder; PTHrP

\section{Introduction}

Through alternate splicing, the multi-exonic PTHrP gene gives rise to three initial translation products: PTHrP(1-139), PTHrP(1-141), and PTHrP(1-173). These three peptides are identical in amino acid

*Correspondence: S Vaidyanathan, Regional Spinal Injuries Centre, District General Hospital, Town Lane, Southport, Merseyside PR8 6PN, UK sequence $(1-139)$ and differ only in their carboxy termini. The three initial PTHrP translation products undergo extensive post-translational processing and give rise to a family of mature secretory forms of the peptide, each with its own physiological function(s). Wysolmerski and Stewart ${ }^{1}$ stated that the functions or physiological roles of PTHrP can be broadly divided into four categories. First, the aminoterminal PTHrP 
stimulates transepithelial calcium transport in a variety of tissues, including the renal tubule, the placenta, and the mammary gland. Second, PTHrP is a smooth muscle relaxant in a broad range of tissues, including uterus, urinary bladder, stomach, small intestine, colon, and the arterial wall. In each of these tissues, PTHrP is upregulated by mechanical stretch. Third, PTHrP is a regulator of cellular differentiation. The effects of PTHrP on cell proliferation and apoptosis may well be indirect, and mediated through its effects on cell differentiation. Finally, disruption of the PTHrP gene or its receptor is lethal in fetal or immediate postpartum life, indicating that PTHrP is an important developmental factor.

PTHrP is produced in the smooth muscle cells of the urinary bladder. Obstruction of bladder outflow leads to an increase in PTHrP mRNA expression, whereas persistent maintenance of bladder in the empty state through either ureteral diversion or placement of an indwelling catheter results in a sustained lowering of PTHrP mRNA levels in the bladder wall. These observations suggest a possible physiological role for PTHrP in the regulation of bladder smooth muscle tone. Histochemical studies performed on distended bladder tissue indicated the presence of PTHrP immunoreactivity in smooth muscle cells. ${ }^{2}$ Philbrick and associates ${ }^{3}$ stated that the role of PTHrP in the vesical transitional epithelium is not known.

Persons with spinal cord injury (SCI) and neuropathic bladder often suffer from cystitis. The lumenal surface of the bladder is lined by a layer of superficial umbrella cells that deposit on their apical surfaces a quasi-crystalline array of hexagonal complexes made up of four integral membrane glycoproteins known as uroplakins. In vitro binding assays have shown that two of the uroplakins, UPIa and UPIb, can specifically bind to uropathogenic Escherichia coli expressing type 1 pili. The consequence of bacterial colonisation of the normally-innervated urinary bladder is exfoliation and excretion of the infected and damaged cells. This process of cell elimination is proposed to be an innate host defence mechanism of the urinary tract. Bacterial attachment results in massive exfoliation of the terminally differentiated superficial cells of the transitional epithelium, thus exposing the underlying, less differentiated urothelial cells. Exfoliation occurs through a rapid apoptosis-like mechanism involving caspase (cysteine-containing aspartate-specific proteases) activation, and host DNA fragmentation as revealed by terminal deoxytransferase-mediated deoxyuridine triphosphate nick end labeling (TUNEL) assays with frozen infected mouse bladder sections. The speed with which DNA fragmentation occurs after inoculation suggests that urothelial cells may be sensitised to undergo rapid programmed cell death (apoptosis) in response to infection by type 1-piliated pathogens. Given that PTHrP (containing sequence of amino acids $87-107$, which represents the putative nucleolar targeting signal) can inhibit apoptosis in chondrocytic cells, we planned a comparative study of immunohistochemical staining using antibodies raised against human PTHrP (43-52), and PTHrP (127-138), of the vesical transitional epithelium of SCI patients, and able-bodied individuals with intact neuraxis. We discuss the concept of apoptosis of urothelium in the pathogenesis of cystitis, and how PTHrP plays a role in the mechanism of apoptosis. By demonstrating the presence of PTHrP in the transitional epithelium of neuropathic bladder, we hypothesise that PTHrP may play a role in the urothelial apoptosis, and thereby, in the genesis of cystitis in SCI patients.

\section{Patients and methods}

This research project was approved by the North Sefton Research Ethics Committee. The criteria for inclusion were: (1) Adult male patients with SCI who are registered with the Regional Spinal Injuries Centre, Southport, England; (2) Patients should have neuropathic bladder due to spinal cord injury sustained at least ten years ago; (3) Patients should be undergoing a therapeutic procedure in the urinary tract such as endoscopic removal of bladder stone, insertion of ureteric stent, etc; (4) The SCI patients should not be suffering from acute urinary infection; and (5) Patients willing to give written informed consent for undergoing biopsy of the urinary bladder after having read the patient information sheet, and having had an opportunity to discuss any of their concerns with the attending doctor from the spinal unit.

Cold cup biopsy was taken from the trigone of the urinary bladder. Most of the patients required either regional or inhalational anaesthesia in order to control spasms, and to block nociceptive stimuli arising from the bladder and urethra which could possibly trigger autonomic dysreflexia in susceptible patients. After taking mucosal biopsies, the biopsy sites were fulgurated with diathermy to prevent any bleeding. Indwelling urethral catheter drainage was maintained for a minimum of $24 \mathrm{~h}$. The patients stayed in the hospital for at least $24 \mathrm{~h}$. All patients received intravenous antibiotic (usually $160 \mathrm{mg}$ of gentamicin) prior to the procedure. Subsequently, the patients were prescribed antibacterials (usually trimethoprim $200 \mathrm{mg}$ 12-hourly) by mouth for 5 days.

The control specimens were bladder biopsies taken from ten able-bodied individuals who did not have malignancy of the urinary bladder. The paraffin blocks of bladder biopsies of control subjects were taken from the archives of the pathology department of Southport District General Hospital. The histopathology of each bladder biopsy was recorded by examining sections stained with haematoxylin and eosin. Only those bladder biopsies which contained transitional epithelium were included in this comparative immunohistochemical study. Therefore, tissue sections which showed only squamous epithelium, or those biopsy specimens in which the urothelium had been denuded because of surgical trauma were excluded from analysis. 


\section{Immunohistochemistry for PTHrP}

The biopsy specimens from patients were fixed in buffered formalin $10 \%$ and then embedded in Paraplast. Five micron sections were cut and fixed to Vectabond-coated slides. The sections were deparaffinised by incubation at $60^{\circ} \mathrm{C}$ for $1 \mathrm{~h}$ followed by two 10 min treatments in xylene. The sections were rehydrated by two 10-min incubations in each $100 \%, 90 \%$ and $70 \%$ ethanol. The sections were then rinsed with $0.01 \mathrm{~mol} / 1$ phosphate-buffered saline (PBS) (pH 7.4). Thereafter, the slides were treated with $0.1 \%$ hydrogen peroxide in PBS for $30 \mathrm{~min}$ to quench endogenous peroxidase activity. The slides were then rinsed with PBS. The sections were covered with normal swine serum for $30 \mathrm{~min}$ at room temperature to block nonspecific binding, then drained without rinsing. The slides were not washed. The first antibody was a rabbit antiPTHrP (43-52), generated to a synthetic peptide representing the amino acid sequence of $43-52$ of the human PTHrP molecule. In a parallel set of experiments, the first antibody was a rabbit anti-PTHrP $127-$ 138 , raised to a synthetic peptide representing the amino acid sequence of 127-138 of the human PTHrP. These antibodies were used in a dilution of 1 in 100 . For each section, a negative control of bladder biopsy was included. This negative control section was treated with normal rabbit serum in a strength of 1 in 100 in PBS instead of PTHrP antibody. After a period of $60 \mathrm{~min}$, the first antibody or the normal rabbit serum was washed with PBS three times for 5 min each, with agitation. Thereafter, the second antibody was added in a strength of 1 in 100 . The second antibody was peroxidase-conjugated, affinity-isolated, goat, antirabbit immunoglobulins, mainly IgG. The sections were incubated for $40 \mathrm{~min}$ at room temperature. Subsequently, the slides were washed with PBS three times for $5 \mathrm{~min}$ each, with agitation. The slides were then treated with Sigma FAST DAB which was newly prepared for each batch of sections. One tablet of urea hydrogen peroxide and one tablet of 3,3-diaminobenzidine tetrahydrochloride were dissolved in one $\mathrm{ml}$ of distilled water. The tissue sections were immersed with the freshly prepared, and ready-to-use, DAB solution which was added to the sections by a pipette. After a period of $2 \mathrm{~min}$, the slides were rinsed with distilled water. The sections were then counterstained with haematoxylin, differentiated with $0.1 \%$ hydrochloric acid, and blued up with tap water. The sections were dehydrated by consecutive 2 -min rinses in $70 \%, 90 \%$ and $100 \%$ ethanol, followed by immersion in xylene. The sections were mounted with DPX and coverslipped. The bladder biopsy slides were examined for immunostaining of transitional epithelium. A batch of skin sections were stained in the above manner. These skin tissue sections acted as positive controls for PTHrP.

\section{Results}

The skin sections showed positive antibody staining against PTHrP $(43-52)$ and PTHrP $(127-138)$ by the keratinocytes. The dermal stroma showed only isolated foci of PTHrP staining due to occasional smooth muscle cells and pericytes. The transitional epithelium in the bladder biopsy sections taken from ten ablebodied individuals without history of vesical malignancy, showed no immunostaining using either of the PTHrP antibodies. Only inflammatory cells and stroma showed positive immunostaining in these sections. Of the 14 biopsies of SCI patients, positive immunostaining with both antibodies was found in four cases; five biopsies showed no immunostaining with either of the antibodies; and five biopsies showed positive immunostaining only against PTHrP $(43-52)$. Positive staining to PTHrP (43-52) was seen mainly associated with cell membrane of the superficial cells (Figure 1). Positive immunostaining to PTHrP $(127-138)$ was also observed predominantly in the superficial layers of the transitional epithelium, and was mainly cytoplasmic (Figure 2).

\section{Discussion}

Several growth factors and cytokines have been shown to modulate PTHrP production. As these substances are often produced in the same tissues as PTHrP, it is likely that unique paracrine loops exist in each tissue to control PTHrP expression. PTHrP may modulate cellular function in a dual mode of action: first, by binding and activating its cognate cell surface Gprotein-coupled receptor and, second, by direct intracellular effects following translocation to the nucleus and/or nucleolus of the target cell. ${ }^{5}$ PTHrP can be secreted by either the regulated secretory pathway, or the constitutive secretory pathway. The cellular response to PTHrP-nuclear interaction is cell-

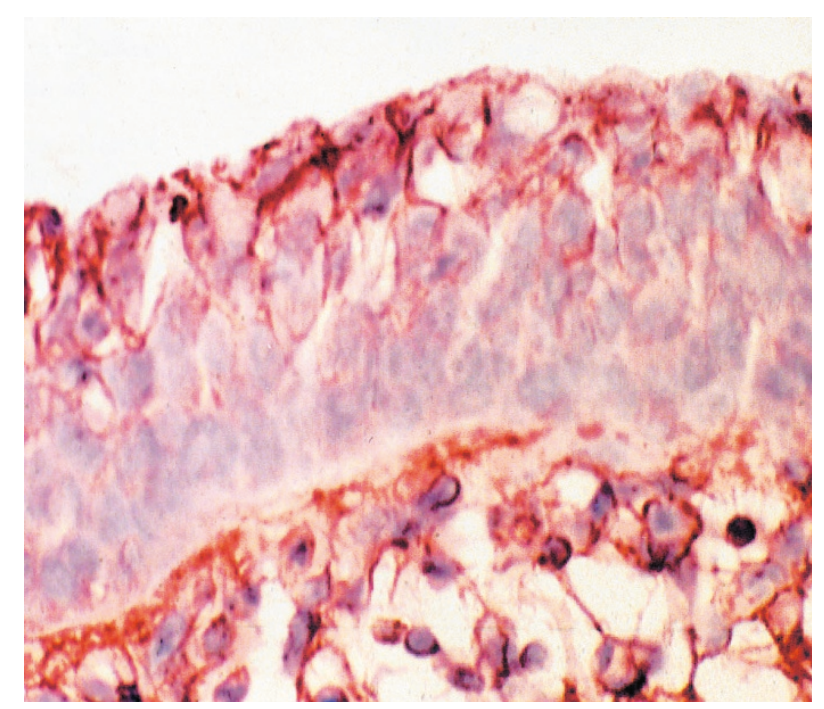

Figure 1 Urinary bladder biopsy-immunohistochemical staining with antibody to PTHrP (43-52). Positive staining was seen mainly associated with cell membrane of the superficial transitional cells 


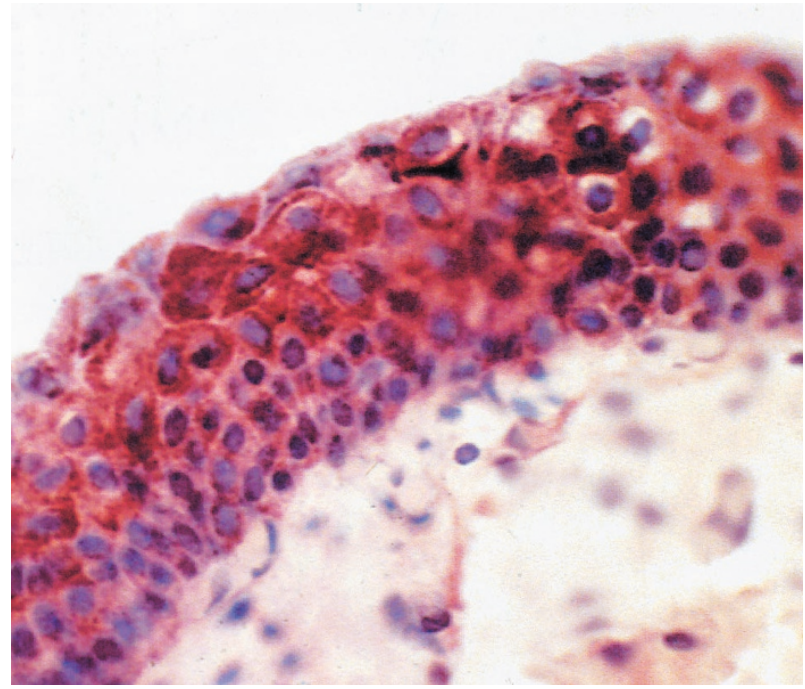

Figure 2 Urinary bladder biopsy-immunohistochemical staining with antibody to PTHrP (127-138). Positive immunostaining was observed predominantly in the superficial layers of the transitional epithelium, and was mainly cytoplasmic

specific, in some cell types preventing apoptosis, in others inhibiting proliferation, and in still other cell types, such as vascular smooth muscle, activating the cell cycle. ${ }^{6}$

PTHrP has been implicated as an autocrine modulator of growth and differentiation in the colon. ${ }^{7}$ When a monoclonal antibody directed against epitopes between amino acids 53-64 which share no homology to PTH was used, $94.3 \%$ of the tissue samples of normal colon were negative. In contrast, $91.5 \%$ of the samples from colon cancer stained positive for PTHrP. Experimental evidence suggests a possible role for PTHrP in growth and differentiation of intestinal epithelial cells. Using rabbit antiserum either to a recombinant human PTHrP ( -5 to 139) or to synthetic PTHrP (1-34), PTHrP immunoreactivity was observed in rat jejunal epithelial cells all along the villus, but staining was absent from crypt cells. ${ }^{8}$ Expression of the peptide in villus epithelium but not in crypt cells suggested a role in growth or differentiation of gastrointestinal epithelial cells. By fluorescence immunocytochemistry, Lam and associates ${ }^{9}$ demonstrated PTHrP staining in all asynchronously cycling $\mathrm{HaCaT}$ cells, with stronger staining in cells that were undergoing division. PTHrP was localised to the nucleolus in the quiescent $\left(\mathrm{G}_{1}\right)$ cell population, where evidence indicates that PTHrP may be involved in delaying apoptosis. Expression of PTHrP forms containing the nucleolar targeting signal prolonged survival of chondrocytic cells under conditions that promoted programmed cell death. The amino acids $87-107$ of PTHrP probably represent the putative nucleolar targeting signal. ${ }^{10}$ PTHrP increases the expression of $\mathrm{Bcl}-2$, a protein that controls programmed cell death in chondrocytes. The gene for PTHrP lies upstream of the anti-apoptosis gene product $\mathrm{Bcl}-2$ and by increasing $\mathrm{Bcl}-2$ expression both in vitro and in vivo, evidence was provided that PTHrP is involved in the control of apoptosis. ${ }^{11}$

This study shows the presence of immunoreactivity in the transitional epithelium of the neuropathic bladder of patients with SCI to PTHrP (43-52) in nine out of 14 biopsies, and to PTHrP (127-138) in four out of 14 biopsies taken from patients who had sustained spinal cord injury at least ten years ago. It has been postulated that denervation of the urinary bladder initiates a cascade of events affecting urothelial proliferation, maturation, and apoptosis. ${ }^{12}$ PTHrP acts mainly as a regulator of cellular differentiation. ${ }^{13}$ The effects of PTHrP on urothelial proliferation and apoptosis may well be indirect, and mediated through effects on differentiation. In patients with spinal cord injury and neuropathic bladder, it is possible that the urothelial cell layers which normally express PTHrP during the differentiation of the bladder have been stimulated to grow by the denervation. During this process of 're-differentiation', a more immature epithelium becomes prominent, and is less susceptible to apoptosis as a result of its expression of PTHrP.

A caspase-mediated apoptosis-like mechanism results in the shedding of urothelial cells in response to bladder infection. ${ }^{4}$ In the present study, we demonstrated the presence of immunoreactivity in the transitional epithelium of the neuropathic bladder of patients with SCI to PTHrP (43-52) in nine out of 14 biopsies, and to PTHrP $(127-138)$ in four out of 14 biopsies. No immunostaining was observed in the transitional epithelium of biopsies of control subjects with normally innervated urinary bladder and no history of vesical malignancy. Based on these observations, we propound a role for PTHrP in the pathogenesis of cystitis in SCI patients. Type 1-piliated uropathogens induce programmed cell death and exfoliation of bladder epithelial cells. If PTHrP inhibits programmed cell death (apoptosis) of the epithelial cells lining the lumenal surface of the bladder, PTHrP may increase the susceptibility for cystitis in SCI patients, as eukaryotic cells undergo apoptosis as a means of hindering the progression of an invading pathogen. Further investigations are warranted to study the role of PTHrP in the differentiation and apoptosis of vesical urothelium and thereby, in the pathogenesis of cystitis in SCI patients.

Another area worth investigating is whether PTHrP $(43-52)$, and PTHrP $(127-138)$ will serve as immunohistochemical markers of denervation of the urinary bladder, as transitional epithelium of the normally innervated urinary bladder of subjects without a history of vesical malignancy showed no immunoreactivity to either of these peptides. Spinal cord repair, an innovative treatment for spinal cord injury which is on the horizon, may involve the 
infusion of IN-1 to the injury site (IN-1 is a monoclonal antibody which neutralises NI-250, one of the major inhibitory molecules of myelin); and expansion in vitro of Schwann cells from a patient, followed by grafting back into the injury site. ${ }^{14}$ If PTHrP (43-52) and PTHrP (127-138) are proved to be markers of denervation of the urinary bladder, the effectiveness of new treatment methods for spinal cord injury can be assessed by studying PTHrP expression in the vesical transitional epithelium of biopsy specimens in SCI patients taken at the bed-side with a flexible cystoscope. Thus immunostaining for PTHrP $(43-52)$ and PTHrP $(127-138)$ in bladder biopsies may prove to be a new investigational tool for studying denervation and re-innervation of the urinary bladder in SCI patients analogous to the use of other established indicators of neural innervation such as nerve growth factor (NGF), and growthassociated protein-43 (GAP-43). ${ }^{15}$

\section{Acknowledgements}

Our gratitude to Dr William Philbrick, Section of Endocrinology, Department of Internal Medicine, Yale University School of Medicine, New Haven, Connecticut 06520-8020, USA for reviewing the manuscript, and for thought provoking discussions over the telephone. We are indebted to Dr Philbrick for his valuable comments which we incorporated in this revised version. We thank Mrs Enid Swift, Librarian, Hanley Library, Southport and Formby NHS Trust for providing references.

\section{References}

1 Wysolmerski JJ, Stewart AF. The physiology of parathyroid hormone-related protein: an emerging role as a developmental factor. Ann Rev Physiol 1998; 60: $431-460$.
2 Yamamoto M, Harm SC, Grasser WA, Thiede MA. Parathyroid hormone-related protein in the rat urinary bladder: a smooth muscle relaxant produced locally in response to mechanical stretch. Proc Natl Acad Sci USA 1992; 89: 5326-5330.

3 Philbrick WM et al. Defining the roles of parathyroid hormonerelated protein in normal physiology. Physiol Rev 1996; 76: $127-$ 173

4 Mulvey MA et al. Induction and evasion of host defenses by type 1-piliated uropathogenic Escherichia coli. Science 1998; 282: $1494-1497$.

5 Nguyen MTA, Karaplis AC. The nucleus: a target site for parathyroid hormone-related peptide (PTHrP) action. J Cell Biochem 1998; 70: 193 - 199

6 Massfelder $\mathrm{T}$ et al. Opposing mitogenic and anti-mitogenic actions of parathyroid hormone-related protein in vascular smooth muscle cells: a critical role for nuclear targeting. Proc Natl Acad Sci USA 1997; 94: 13630-13635.

7 Malakouti $\mathrm{S}$ et al. Parathyroid hormone-related protein expression in the human colon: immunohistochemical evaluation. Am Surg 1996; 62: $540-545$.

$8 \mathrm{Li} \mathrm{H}$ et al. Widespread expression of the parathyroid hormonerelated peptide and $\mathrm{PTH} / \mathrm{PTHrP}$ receptor genes in intestinal epithelial cells. Lab Invest 1995; 73: $864-870$.

9 Lam MHC et al. PTHrP and cell division: Expression and localization of PTHrP in a keratinocyte cell line (HaCaT) during the cell cycle. J Cell Physiol 1997; 173: 433-446.

10 Henderson JE et al. Nucleolar localization of parathyroid hormone-related peptide enhances survival of chondrocytes under conditions that promote apoptotic cell death. Mol Cell Biol 1995; 15: $4064-4075$.

11 Amling $\mathrm{M}$ et al. Bcl-2 lies downstream of parathyroid hormonerelated peptide in a signaling pathway that regulates chrondrocyte maturation during skeletal development. J Cell Biol 1997; 136: $205-213$.

12 Vaidyanathan $\mathrm{S}$ et al. Possible role of denervation-induced changes in the pathophysiology of cystitis in patients with spinal cord injury: a hypothesis. Spinal Cord 1997; 35: 708 - 709 .

13 Foley $\mathbf{J}$ et al. PTHrP regulates epidermal differentiation in adult mice. J Invest Dermatol 1998; 111: $1122-1128$.

14 Fawcett JW. Spinal cord repair: from experimental models to human application. Spinal Cord 1998; 36: 811-817.

15 Wakabayashi Y, Maeda T, Aimi Y, Kwok YN. Increase of lowaffinity neurotrophin receptor $\mathrm{p}^{75}$ and growth-associated protein-43 immunoreactivities in the rat urinary bladder during experimentally induced nerve regeneration. J Urol 1998; 160: $1513-1517$ 